\title{
Susceptible supply limits the role of climate in the early SARS-CoV-2 pandemic
}

\author{
Rachel E. Baker ${ }^{1,2 *}$, Wenchang Yang ${ }^{3}$, Gabriel A. Vecchi ${ }^{1,3}$, C. Jessica E. Metcalf ${ }^{2,4}$, Bryan T. Grenfell ${ }^{2,4,5}$ \\ 1Princeton Environmental Institute, Princeton University, Princeton, NJ, USA. ²Department of Ecology and Evolutionary Biology, Princeton University, Princeton, NJ, USA. \\ ${ }^{3}$ Department of Geosciences, Princeton University, Princeton, NJ, USA. ${ }^{4}$ Woodrow Wilson School of Public and International Affairs, Princeton University, Princeton, NJ, \\ USA. ${ }^{5}$ Division of International Epidemiology and Population Studies, Fogarty International Center, National Institutes of Health, Bethesda, MD, USA. \\ *Corresponding author. Email: racheleb@princeton.edu
}

Preliminary evidence suggests that climate may modulate the transmission of SARS-CoV-2. Yet it remains unclear whether seasonal and geographic variations in climate can substantially alter the pandemic trajectory, given high susceptibility is a core driver. Here, we use a climate-dependent epidemic model to simulate the SARS-CoV-2 pandemic probing different scenarios based on known coronavirus biology. We find that while variations in weather may be important for endemic infections, during the pandemic stage of an emerging pathogen the climate drives only modest changes to pandemic size. A preliminary analysis of non-pharmaceutical control measures indicates that they may moderate the pandemic-climate interaction via susceptible depletion. Our findings suggest, without effective control measures, strong outbreaks are likely in more humid climates and summer weather will not substantially limit pandemic growth.

The SARS-CoV-2 pandemic represents an unprecedented public health, social and economic challenge. Sustained local transmission is present in multiple countries, in all continents, and the implications in terms of morbidity and mortality are expected to be severe $(1,2)$. The role of seasonal and geographic climate variations in modulating the transmission of the virus has received increasing attention. Studies using a regression framework have found a role for temperature, relative and specific humidity in the transmission of SARS-CoV-2 (3-7), suggesting that cold, dry conditions increase the transmission of the virus. However, with limited data on the current epidemic, these early-stage results are inevitably inconclusive. Furthermore, the relative importance of climate drivers when compared to high population susceptibility during the pandemic stage of an emerging infection such as SARS-CoV-2, has not been fully characterized.

Climate affects the transmission of several directly-transmitted pathogens (8). Specific humidity (the mass of water vapor in a unit mass of moist air) has been shown to be important for influenza transmission in both laboratory settings (9-11), and in population-level studies (12). Respiratory syncytial virus (RSV), a childhood pathogen, has also been found to be dependent on specific humidity (13) and exhibits latitudinal correlations with climate (14). For both influenza and RSV, low specific humidity increases transmission and epidemics tend to peak in the wintertime in northern latitudes. However, other directly-transmitted infections exhibit different patterns (15), with enteroviruses, for instance, often peaking in the summer months (16).

Prior work on climate and directly-transmitted diseases has typically considered endemic infections, such as seasonal influenza or RSV. Emerging pathogens, in contrast, have distinct dynamics driven by high population susceptibility (17). A key question is the extent to which seasonal and geographic climate variations are relevant in the pandemic phase of an emerging infection. Here we build on known features of endemic human coronaviruses and other directly-transmitted infections to probe this question. Although we do not yet know the climate sensitivity of SARS-CoV-2 transmission directly, data exists on four other coronaviruses that currently circulate in human populations. Two of these coronaviruses, $\mathrm{HCoV}-\mathrm{HKU} 1$ and HCoV-OC43, are of the same betacoronavirus genus as SARS-CoV-2 (18).

We use data on $\mathrm{HCoV}-\mathrm{HKU} 1$ and $\mathrm{HCoV}-\mathrm{OC} 43$ from US census regions to understand the potential climate dependence of betacoronavirus transmission (19). We fit a Susceptible-Infected-Recovered-Susceptible (SIRS) model to case data of $\mathrm{HCoV}-\mathrm{HKU} 1$ and $\mathrm{HCoV}-\mathrm{OC} 43$ where the fitted parameters include the climate dependence of transmission and the length of immunity following infection. All other parameters are fixed, based on values from Kissler et al. 2020 (18). Motivated by the climate-dependence of influenza and RSV, we posit that transmission depends on specific humidity: we use population-weighted average climatology of specific humidity over 2014-2020 taken from the ERA5 reanalysis dataset (20), with population data from (21). We note that specific humidity is dependent on temperature via the ClausiusClapeyron relation and results using both variables have been found to be equivalent for other diseases (13). After fitting the model parameters, we run simulations of the SARS-CoV-2 pandemic under three scenarios. In the first scenario we assume SARS-CoV-2 has the same sensitivity to climate as 
influenza, based on a prior model from laboratory studies $(9$, 12). In the second and third scenarios, we assume SARS-CoV2 has the same climate dependence and length of immunity as $\mathrm{HCoV}-\mathrm{OC} 43$ and $\mathrm{HCoV}-\mathrm{HKU1}$ respectively. While we assume the climate dependence is the same as these three infections, our simulations use $R_{0}$ based on current estimates of SARS-CoV-2 $(18,22)$.

We first consider the seasonality of the endemic betacoronaviruses. Figure 1 shows the average seasonal pattern of endemic betacoronaviruses, OC43 and HKU1, for different regions in the US. Cases of both diseases increase as specific humidity declines (fig. S1). We therefore assume that to some extent transmission will decline with specific humidity, however, the extent of the decline is yet to be determined. We characterize the link between specific humidity and the transmission of SARS-CoV-2 using plausible estimates derived from the two endemic betacoronaviruses as well as influenza. Figure 1A shows different potential functional forms for the climate-transmission relationship. Changes to specific humidity modulate $R_{0}$ between a maximum wintertime value and a hypothesized lower bound, taken from prior studies $(18,22)$. In the extreme cases, transmission $\left(R_{0}\right)$ either rapidly declines as specific humidity increases or has no relationship with specific humidity. The highlighted influenza relationship is based on laboratory studies using the guinea pig animal model (9-11), and later used to predict influenza epidemics in human populations (12). In this case $R_{0}$ values correspond to SARS-CoV-2 estimates.

The other two scenarios in Fig. 1A correspond to the relationship between climate and OC43 and HKU1 transmission. We evaluate the functional form of this relationship by fitting our climate-driven SIRS model to US case data for the two infections (Fig. 1, B and C, and figs. S2 and S3). Our results (Fig. 1, D and E) suggest a somewhat wide range of climate dependency for the two coronaviruses, with HKU1 having a much steeper response to specific humidity then OC43. Strong seasonal forcing has been linked to biennial outbreaks, as observed for HKU1 (fig. S2), in other respiratory pathogens (13) and implies endemic dynamics driven by herd immunity; however this inference may be complicated by cross-protection from other circulating strains (18). While there is some uncertainty in our estimates, simulating a pandemic outbreak using a range of climate-transmission dependencies allows us to explore a wide plausible range of potential climate effects.

We simulate a pandemic invasion for all locations (Fig. 2, $\mathrm{A}$ and $\mathrm{C}$ ) and focus on the results for nine exemplar cities (Fig. 2, C to E) each with a very distinct mean and seasonal cycle of specific humidity (fig. S4). We stress that these initial simulations explore only the interaction of the epidemic (SIRS) model clockwork and seasonality in transmission; they do not address complexities of demography, control and other environmental factors. In Fig. 2, $\mathrm{C}$ to $\mathrm{E}$, we show the evolution of the simulated pandemic, holding population constant, for northern hemisphere, southern hemisphere and tropical locations. The model assumes the outbreak starts at the same time and no control measures are in place, revealing only the effect of climate on pandemic size and duration. For the northern hemisphere locations, we do not see any substantial difference in pandemic size across all three scenarios, despite very different climates in New York, London and Delhi. In the influenza and HKU1 scenarios, tropical locations experience a more sustained, lower intensity pandemic than the northern hemisphere. These scenarios represent a stronger dependence on climate than $\mathrm{OC} 43$, such that the lack of really dry conditions (low specific humidity) in tropical regions means these locations do not experience the high transmission rates of the higher latitudes. However, the outbreak in the tropical cities remains significant, and factors we do not explore here, such as population density, could further exacerbate the size of the epidemic.

We also simulate the pandemic in a range of southern hemisphere locations (Fig. 2D). We see only minor delays in the peak of southern hemisphere locations relative to the northern hemisphere (Fig. 2B), despite the six-month shift in specific humidity seasonality between the two hemispheres (fig. S4). For the OC43 scenario, pandemics are temporally aligned across all locations and of similar magnitude. A stronger climate response for influenza and HKU1 parameters leads to slight regional differences. It is worth noting that our different scenarios also reflect a range of immunity lengths. The size of the pandemic peak is not affected by changes in immunity length (fig. S11), but the timing of latter stage outbreaks is partially dictated by this parameter. The differential timing of secondary peaks in the influenza and HKU1 scenarios, which have a similar climate-dependence, is driven by this variability.

During the pandemic stage of an emerging pathogen the lack of population immunity, i.e., high susceptibility, is a crucial driver. To illustrate this in the general case, we run our simulation model for different climates (represented by the seasonal range of humidity values a location experiences) and different levels of population susceptibility, using the mean specific humidity and seasonality of New York. Figure 3, A to $\mathrm{C}$, shows the results in terms of the size of the pandemic peak. While humidity range does modulate pandemic size, population susceptibility exhibits a much steeper gradient. For novel pathogens, such as SARS-CoV-2, the proportion of the population susceptible to infection may be close to 1 . To illustrate the potential longer term behavior of the pandemic, we plot a typical pandemic trajectory on the SI phase plane (Fig. 3D). The initial pandemic trajectory (red) is relatively independent of seasonal forcing. This then gives way to the endemic attractor (blue) which oscillates around the equilibrium of 
the unforced model (green). These longer term dynamics show a much stronger signature of seasonal forcing than the initial pandemic phase (figs. S9 and S10).

Figures $3 \mathrm{E}$ and 4 show a preliminary exploration of the impact of non-pharmaceutical control on the epidemic trajectory. In Fig. 3E, we show the SI phase plane where the HKU1 parameters of $R_{0}$ are artificially controlled for a six month period to $R_{0}=1.1$. In this scenario, the control measures result in a moderate reduction in peak incidence as the outbreak is shifted to the summer months, however, high susceptibility still results in a substantial numbers of cases. In Fig. 4, we explore the interaction between the climate and control measures in more detail. We consider four scenarios: climate-dependencies based on OC43 and HKU1 as well as control measures where $R_{0}=1.1$ or 1.3 , representing limited transmission. For each scenario we vary the length of the control measure and the location, however for simplicity we assume all control measures start at two times, four weeks and six weeks after the disease is introduced. We note that these control measures are simplified test cases and do not represent the local heterogeneity and efficacy of current controls, which is yet to be determined. These results show change to peak incidence, changes to number infected are shown in fig. S5.

For all control scenarios we assume a degree of transmission during the control period, such that $R_{0}>1$, resulting in an increase in population immunity over time. In the scenarios where $R_{0}=1.3$, immunity builds faster and control measures work to reduce the pandemic peak after several months. In the $R_{0}=1.1$ scenario more time is required for population immunity to build such that the pandemic peak is reduced across all locations. In this scenario, as susceptibility declines, the climate plays a more substantial role in determining pandemic peak size (Fig. 4, A and B). When $R_{0}=$ 1.1 in both the HKU1 and OC43 scenarios, releasing control measures close to the month of maximum transmission may result in a larger pandemic peak compared to the no control scenario, particularly in the higher latitudes where transmission likely increases in the winter (fig. S6).

More broadly, our simulated control measures imply that the key determinant of reduced peak incidence is the extent to which population immunity builds over the control period, demonstrated by the higher efficacy of the $R_{0}=1.3$ control scenario in mitigating peak incidence. The climate plays a complex role in tuning the efficacy of potential control efforts, resulting in differential outcomes depending on location, however population susceptibility remains a fundamental driver. Further exploration of these complexities may be necessary when evaluating location-specific controls. The timing of introduction and the efficacy of local control measures as well as factors such as population density and contact patterns could also shape future outcomes.
Serological surveys, at the local level, will be important for tracking the build up of immunity over time. Moreover, implementing control measures buys crucial time while vaccine and other treatments are developed.

There are several caveats to interpreting these results. Primarily, these simulations do not address location specific factors such as spatial and social mixing patterns, contact networks, population density and the specifics of control timing and efficacy. In particular, our results apply most closely to relatively well-mixed epidemics in large cities. Rural areas (with potentially lower $R_{0}$; see figs. S7 and S8), may have a more delayed initial epidemic with complex consequences for ensuing interactions with climate drivers. Our model also does not account for potential cross-protection from other coronavirus infections (18). Cross-immunity may contribute to the seasonality of endemic coronaviruses, meaning estimated climate drivers could be even weaker than we suggest and our main findings, conservative $(16,23)$. Finally, results from influenza and RSV suggest that high precipitation may play a role in driving transmission $(13,24)$, particularly in tropical locations. Due to limited data on betacoronaviruses from tropical locations, we have not been able to confirm whether a rainfall signal exists. Precipitation effects and other drivers such as schooling may also impact the epidemic trajectory, particularly post-pandemic. We further test for the sensitivity of our results to changes in core parameter values (materials and methods and figs. S7 to S11). These analyses suggest our results are qualitatively robust to variations in climate dependency and weather fluctuations.

Our results suggest that while climate may play a role in modulating detailed aspects of the size and timescales of a pandemic outbreak within a particular location, population immunity is a much more fundamental driver of pandemic invasion dynamics. Although our HKU1 scenarios presents a modest role for climate in terms of shifting the timing and intensity of the pandemic, a scenario with OC43 parameters is equally likely. In terms of the SARS-CoV-2 pandemic, our results imply that both tropical and temperate locations should prepare for severe outbreaks of the disease and that summertime temperatures will not effectively limit the spread the infection. However, this does not mean the climate is not important in the longer term. Endemic cycles of the disease will likely be tied to climate factors and seasonal peaks may vary with latitude (figs. S3, S9, and S10). A more detailed understanding of climate drivers as well as immunity length will be crucial for understanding the implications of control measures. Furthermore, weather and near-term climate forecasts could be helpful for predicting secondary outbreaks after the initial pandemic phase has passed.

\section{REFERENCES AND NOTES}

1. R. Verity, L. C. Okell, I. Dorigatti, P. Winskill, C. Whittaker, N. Imai, G. CuomoDannenburg, H. Thompson, P. Walker, H. Fu, A. Dighe, J. Griffin, A. Cori, M. 
Baguelin, S. Bhatia, A. Boonyasiri, Z. M. Cucunuba, R. Fitzjohn, K. A. M. Gaythorpe, W. Green, A. Hamlet, W. Hinsley, D. Laydon, G. Nedjati-Gilani, S. Riley, S. vanElsand, E. Volz, H. Wang, Y. Wang, X. Xi, C. Donnelly, A. Ghani, N. Ferguson, Estimates of the severity of COVID-19 disease. medRxiv 2020.03.09.20033357 [Preprint]. 13 March 2020; https://doi.org/10.1101/2020.03.09.20033357.

2. R. Li, C. Rivers, Q. Tan, M. B. Murray, E. Toner, M. Lipsitch, The demand for inpatient and ICU beds for COVID-19 in the US: lessons from Chinese cities. medRxiv 2020.03.09.20033241 [Preprint]. 16 March 2020; https://doi.org/10.1101/2020.03.09.20033241.

3. Q. Bukhari, Y. Jameel, Will coronavirus pandemic diminish by summer? SSRN 3556998 [Preprint]. 17 March 2020; https://dx.doi.org/10.2139/ssrn.3556998.

4. J. Wang, K. Tang, K. Feng, W. Lv, High temperature and high humidity reduce the transmission of COVID-19. SSRN 3551767 [Preprint]. 9 March 2020; https://dx.doi.org/10.2139/ssrn.3551767.

5. M. M. Sajadi, P. Habibzadeh, A. Vintzileos, S. Shokouhi, F. Miralles-Wilhelm, A. Amoroso, Temperature, humidity and latitude analysis to predict potential spread and seasonality for COVID-19. SSRN 3550308 [Preprint]. 5 March 2020; https://dx.doi.org/10.2139/ssrn.3550308.

6. W. Luo, M. S. Majumder, D. Liu, C. Poirier, K. D. Mandl, M. Lipsitch, M. Santillana, The role of absolute humidity on transmission rates of the COVID-19 outbreak. medRxiv 2020.02.12.20022467 [Preprint]. 17 February 2020; https://doi.org/10.1101/2020.02.12.20022467.

7. Y. Ma, Y. Zhao, J. Liu, X. He, B. Wang, S. Fu, J. Yan, J. Niu, B. Luo, Effects of temperature variation and humidity on the mortality of COVID-19 in Wuhan. medRxiv 2020.03.15.20036426 [Preprint]. 18 March 2020; https://doi.org/10.1101/2020.03.15.20036426.

8. R. E. Baker, A. S. Mahmud, C. J. E. Metcalf, Dynamic response of airborne infections to climate change: Predictions for varicella. Clim. Change 148, 547-560 (2018). doi:10.1007/s10584-018-2204-4

9. J. Shaman, M. Kohn, Absolute humidity modulates influenza survival, transmission, and seasonality. Proc. Natl. Acad. Sci. U.S.A. 106, 3243-3248 (2009). doi:10.1073/pnas.0806852106 Medline

10. A. C. Lowen, J. Steel, Roles of humidity and temperature in shaping influenza seasonality. J. Virol. 88, 7692-7695 (2014). doi:10.1128/JVI.03544-13 Medline

11. A. C. Lowen, S. Mubareka, J. Steel, P. Palese, Influenza virus transmission is dependent on relative humidity and temperature. PLOS Pathog. 3, 1470-1476 (2007). doi:10.1371/journal.ppat.0030151 Medline

12. J. Shaman, V. E. Pitzer, C. Viboud, B. T. Grenfell, M. Lipsitch, Absolute humidity and the seasonal onset of influenza in the continental United States. PLOS Biol. 8, e1000316 (2010). doi:10.1371/journal.pbio.1000316 Medline

13. R. E. Baker, A. S. Mahmud, C. E. Wagner, W. Yang, V. E. Pitzer, C. Viboud, G. A. Vecchi, C. J. E. Metcalf, B. T. Grenfell, Epidemic dynamics of respiratory syncytial virus in current and future climates. Nat. Commun. 10, 5512 (2019). doi:10.1038/s41467-019-13562-y Medline

14. V. E. Pitzer, C. Viboud, W. J. Alonso, T. Wilcox, C. J. Metcalf, C. A. Steiner, A. K. Haynes, B. T. Grenfell, Environmental drivers of the spatiotemporal dynamics of respiratory syncytial virus in the United States. PLOS Pathog. 11, e1004591 (2015). doi:10.1371/journal.ppat.1004591 Medline

15. M. E. Martinez, The calendar of epidemics: Seasonal cycles of infectious diseases. PLOS Pathog. 14, e1007327 (2018). doi:10.1371/journal.ppat.1007327 Medline

16. S. Takahashi, Q. Liao, T. P. Van Boeckel, W. Xing, J. Sun, V. Y. Hsiao, C. J. E. Metcalf, Z. Chang, F. Liu, J. Zhang, J. T. Wu, B. J. Cowling, G. M. Leung, J. J. Farrar, H. R. van Doorn, B. T. Grenfell, H. Yu, Hand, foot, and mouth disease in China: Modeling epidemic dynamics of enterovirus serotypes and implications for vaccination. PLOS Med. 13, e1001958 (2016). doi:10.1371/journal.pmed.1001958 Medline

17. J. R. Gog, S. Ballesteros, C. Viboud, L. Simonsen, O. N. Bjornstad, J. Shaman, D. L. Chao, F. Khan, B. T. Grenfell, Spatial transmission of 2009 pandemic influenza in the US. PLOS Comput. Biol. 10, e1003635 (2014). doi:10.1371/journal.pcbi.1003635 Medline

18. S. M. Kissler, C. Tedijanto, E. Goldstein, Y. H. Grad, M. Lipsitch, Projecting the transmission dynamics of SARS-CoV-2 through the postpandemic period. Science eabb5793 (2020). doi:10.1126/science.abb5793 Medline

19. Centers for Disease Control and Prevention, Surveillance for common human coronaviruses www.cdc.gov/surveillance/nrevss/coronavirus/index.html.
20. L. Hoffmann, G. Günther, D. Li, O. Stein, X. Wu, S. Griessbach, Y. Heng, P. Konopka, R. Müller, B. Vogel, J. S. Wright, From ERA-Interim to ERA5: The considerable impact of ECMWF's next-generation reanalysis on Lagrangian transport simulations. Atmos. Chem. Phys. 19, 3097-3124 (2019). doi:10.5194/acp-193097-2019

21. NASA Socioeconomic Data and Applications Center (SEDAC), Gridded Population of the World, Version 4 (GPWv4): Population Density, Revision 11. Palisades, NY; https://sedac.ciesin.columbia.edu/data/set/gpw-v4-population-density-rev11 [accessed March 2020]

22. S. W. Park, B. M. Bolker, D. Champredon, D. J. D. Earn, M. Li, J. S. Weitz, B. T. Grenfell, J. Dushoff, Reconciling early-outbreak estimates of the basic reproductive number and its uncertainty: framework and applications to the novel coronavirus (SARS-CoV-2) outbreak. medRxiv 2020.01.30.20019877 [Preprint]. 28 February 2020; https://doi.org/10.1101/2020.01.30.20019877.

23. M. Kamo, A. Sasaki, The effect of cross-immunity and seasonal forcing in a multistrain epidemic model. Physica D 165, 228-241 (2002). doi:10.1016/S01672789(02)00389-5

24. J. D. Tamerius, J. Shaman, W. J. Alonso, K. Bloom-Feshbach, C. K. Uejio, A. Comrie, C. Viboud, Environmental predictors of seasonal influenza epidemics across temperate and tropical climates. PLOS Pathog. 9, e1003194 (2013). doi:10.1371/journal.ppat.1003194 Medline

25. R. E. Baker, rebaker64/climate-cov: R1. Zenodo (2020): doi:10.5281/zenodo.3787766.

26. M. E. Killerby, H. M. Biggs, A. Haynes, R. M. Dahl, D. Mustaquim, S. I. Gerber, J. T. Watson, Human coronavirus circulation in the United States 2014-2017. J. Clin. Virol. 101, 52-56 (2018). doi:10.1016/j.jcv.2018.01.019 Medline

27. R. Gelaro, W. McCarty, M. J. Suárez, R. Todling, A. Molod, L. Takacs, C. Randles, A. Darmenov, M. G. Bosilovich, R. Reichle, K. Wargan, L. Coy, R. Cullather, C. Draper, S. Akella, V. Buchard, A. Conaty, A. da Silva, W. Gu, G.-K. Kim, R. Koster, R. Lucchesi, D. Merkova, J. E. Nielsen, G. Partyka, S. Pawson, W. Putman, M. Rienecker, S. D. Schubert, M. Sienkiewicz, B. Zhao, The Modern-Era Retrospective Analysis for Research and Applications, Version 2 (MERRA-2). J. Clim. 30, 54195454 (2017). doi:10.1175/JCLI-D-16-0758.1 Medline

28. L. Bao, W. Deng, H. Gao, C. Xiao, J. Liu, J. Xue, Lv. Qi, J. Liu, P. Yu, Y. Xu, F. Qi, Y. Qu, F. Li, Z. Xiang, H. Yu, S. Gong, M. Liu, G. Wang, S. Wang, Z. Song, Y. Liu, W. Zhao, Y. Han, L. Zhao, X. Liu, Q. Wei, C. Qin, Lack of reinfection in rhesus macaques infected with SARS-CoV-2. bioRxiv 2020.03.13.990226 [Preprint]. 1 May 2020; https://doi.org/10.1101/2020.03.13.990226.

29. K. A. Callow, H. F. Parry, M. Sergeant, D. A. Tyrrell, The time course of the immune response to experimental coronavirus infection of man. Epidemiol. Infect. 105 435-446 (1990). doi:10.1017/S0950268800048019 Medline

\section{ACKNOWLEDGMENTS}

Funding: REB is supported by the Cooperative Institute for Modelling the Earth System (CIMES). WY is supported by Princeton Environmental Institute/Princeton Institute for International and Regional Studies. Author contributions: Conceptualization: REB, WY, GAV, CJEM, BTG; Data curation: REB, WY; Formal analysis: REB; Methodology: REB, WY, GAV, CJEM, BTG; Software: REB, WY, GV; Visualization: REB, GV; Writing, original draft: REB; Writing, reviewing and editing: REB, WY, GAV, CJEM, BTG. Competing interests: The authors declare no competing interests. Data and materials availability: US coronavirus data are available from the The National Respiratory and Enteric Virus Surveillance System (NREVSS) on request:

https://www.cdc.gov/surveillance/nrevss/coronavirus/index.html . Climate data are publicly available from ERA5 and NASA; ERA5 data: https://www.ecmwfint/en/forecasts/datasets/reanalysis-datasets/era5 and NASA MERRA data: https://gmao.gsfc.nasa.gov/reanalysis/MERRA/. Code for running the model is available via github/rebaker64/climate-cov (25). This work is licensed under a Creative Commons Attribution 4.0 International (CC BY 4.0) license, which permits unrestricted use, distribution, and reproduction in any medium, provided the original work is properly cited. To view a copy of this license, visit https://creativecommons.org/licenses/by/4.0/. This license does not apply to figures/photos/artwork or other content included in the article that is credited to a third party; obtain authorization from the rights holder before using such material. 


\section{SUPPLEMENTARY MATERIALS}

science.sciencemag.org/cgi/content/full/science.abc2535/DC1

Materials and Methods

Figs. S1 to S11

References (26-29)

MDAR Reproducibility Checklist

15 April 2020; accepted 14 May 2020

Published online 18 May 2020

$10.1126 /$ science.abc2535 

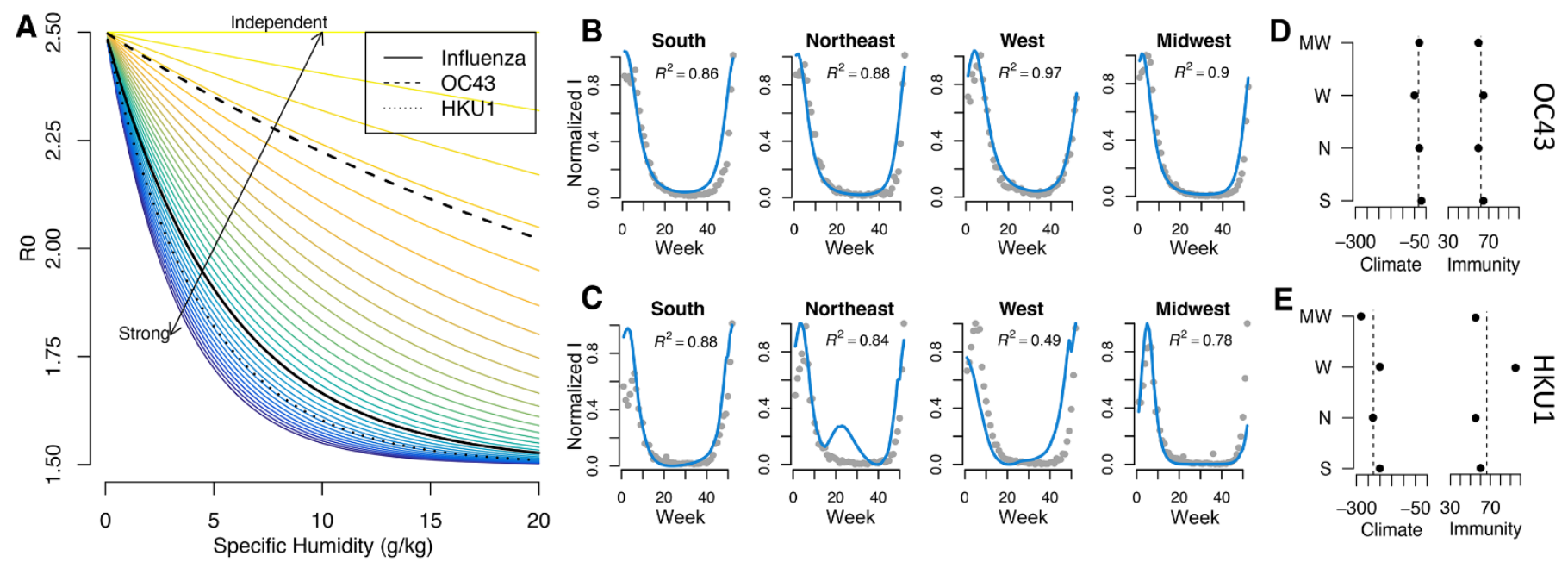

Fig. 1. Specific humidity and transmission. (A) Colored lines represent different hypotheses for the relationship between climate and transmission for SARS-CoV-2. Values of $R_{0}$ reflect SARS-CoV-2 estimates and the functional climate-dependence of influenza transmission, OC43 transmission and HKU1 transmission is shown with black lines. (B and $C$ ) A summary of seasonal model fits (blue line) for scaled average weekly cases (grey) of OC43 (B) and HKU1 (C) (our model captures the biennial cycles of HKU1, shown in fig. S3 and detailed model fits for OC43 shown in fig. S2). $R^{2}$ values are shown inset. ( $D$ and $E$ ) Fit results in terms of climate-dependence and immunity length (weeks) for OC43 (D) and HKU1 (E), where mean fits are shown with dashed lines. 
A
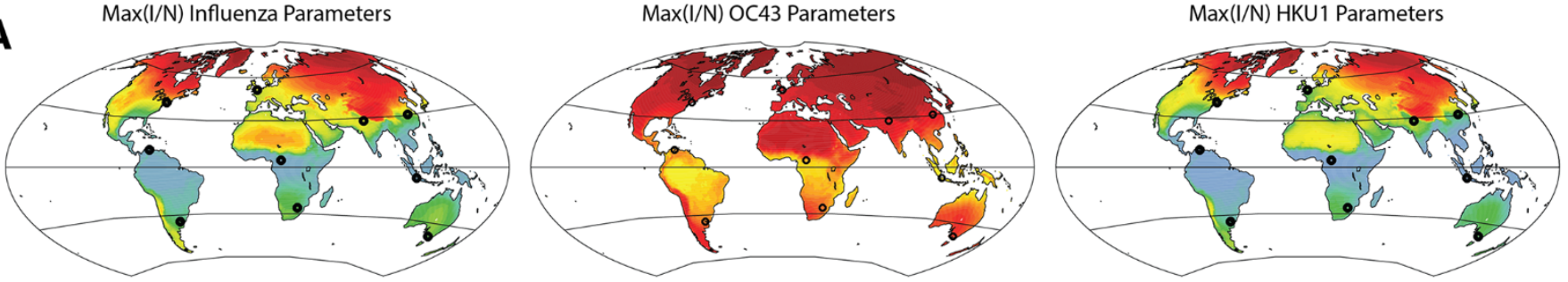

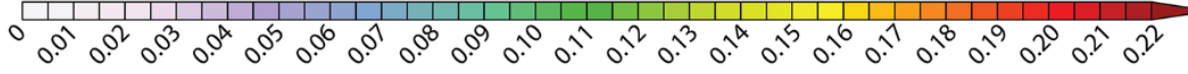

B
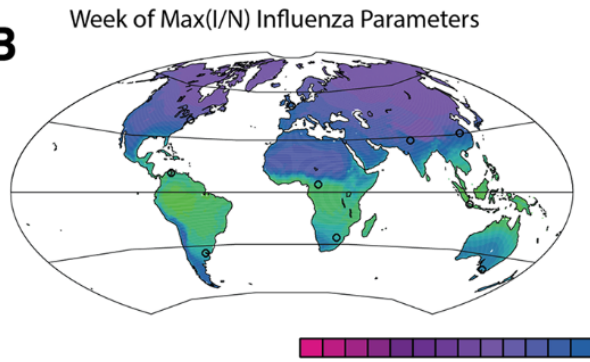

Week of Max(I/N) OC43 Parameters

Week of Max $(\mathrm{I} / \mathrm{N})$ HKU1 Parameters
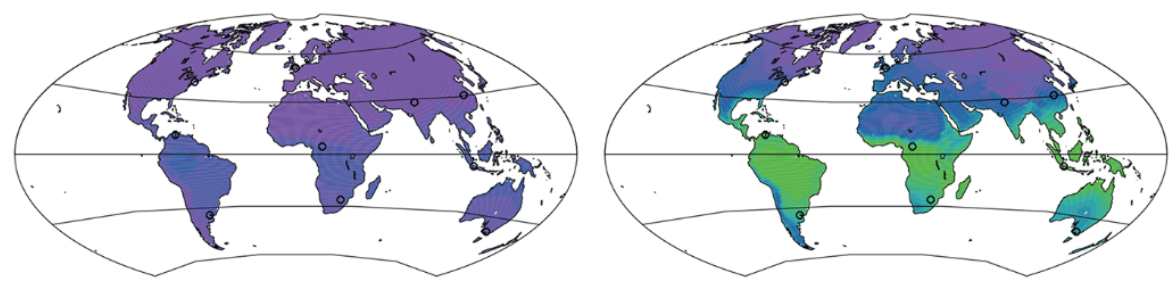

C
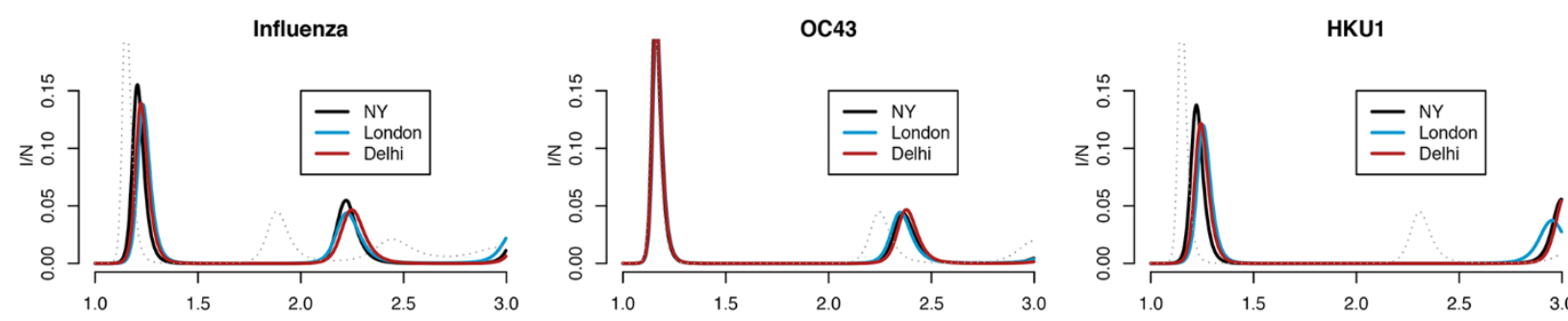

D
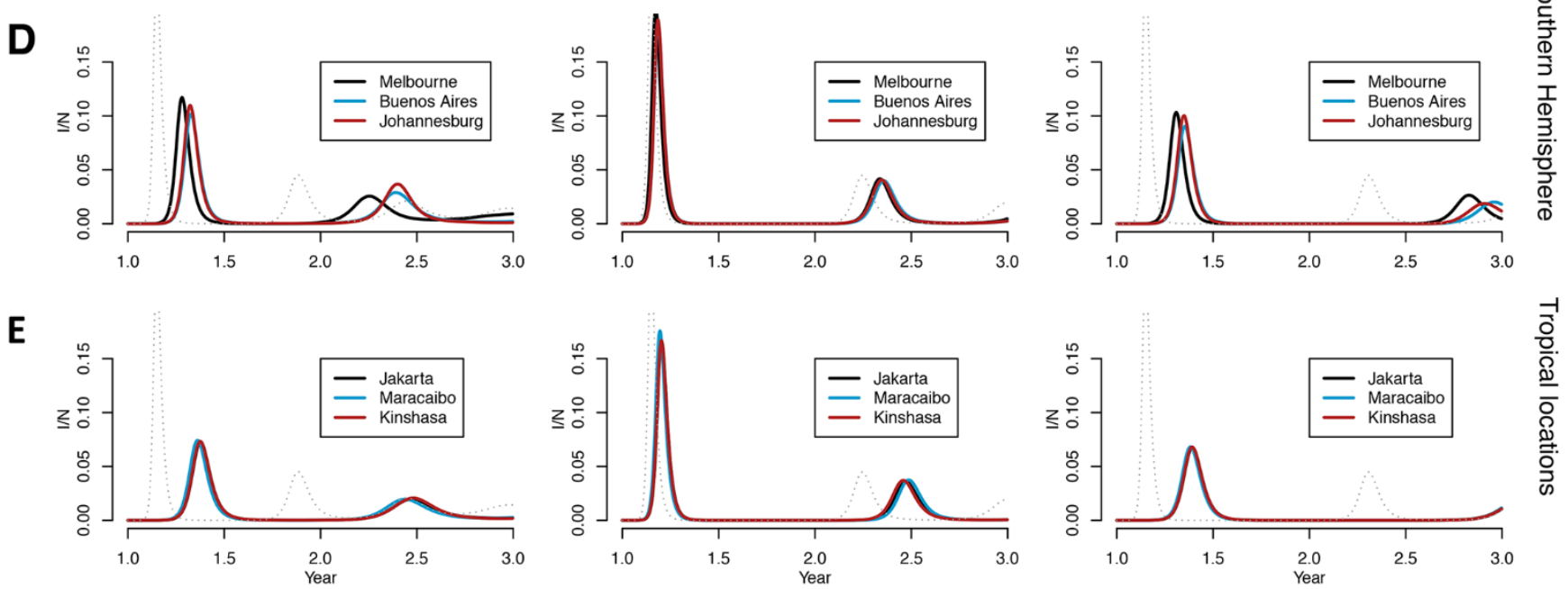

Fig. 2. Global model results and nine example trajectories. (A and B) Maximum I/N (A) and the timing of peak incidence (B) for global locations. Black circles show locations where trajectories are explicitly shown. (C to E) Simulated pandemics are shown for cities in the northern hemisphere (C), the southern hemisphere (D), and tropical locations (E). The dotted line represents a pandemic with no climate dependence. 
A Influenza params, peak $\mathrm{V} / \mathrm{N}$

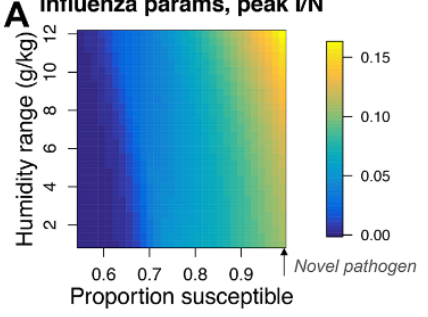

B OC43 params, peak I/N I/N

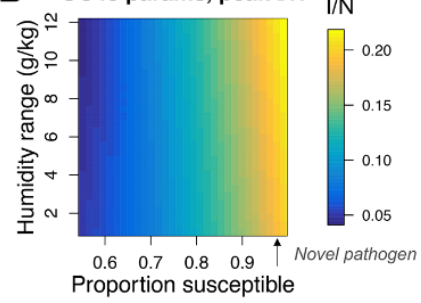

C HKU1 params, peak $\mathrm{I} / \mathrm{N}$

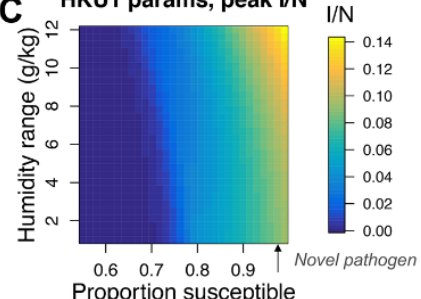

D
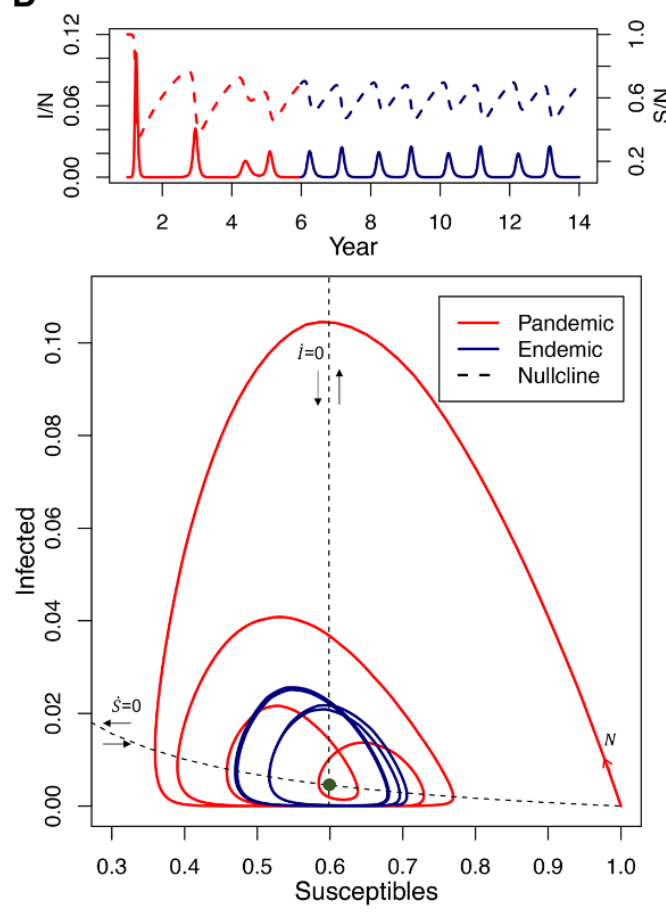

E
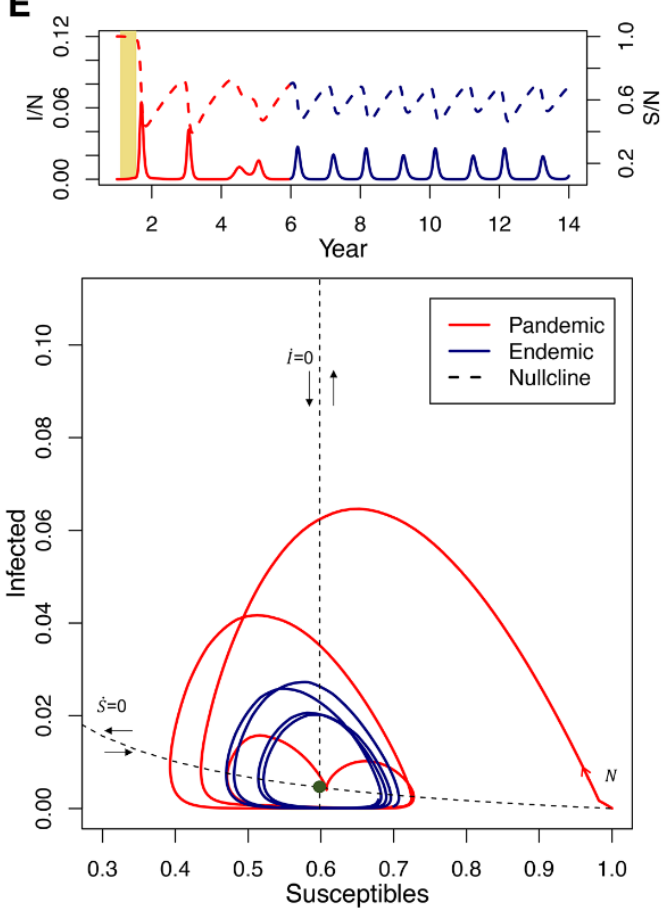

Fig. 3. Pandemic peak size depends on proportion susceptible. (A to $C$ ) For the three scenarios, influenza (A), OC43 (B), and HKU1 (C), the surface plot shows the dependence of maximum pandemic incidence/capita on seasonal range of humidity and proportion of population susceptible, assuming mean humidity of New York. (D) The time series from pandemic to endemic outbreaks for an example location (Wuhan with HKU1 params) (top) and the equivalent SI phase plane of pandemic and epidemic trajectories (bottom). The two nullclines are from the unforced SIRS using mean $R_{0}$. The green circle represents the equilibrium of the unforced model. (E) The same trajectory but with a six month control period (reducing $R_{0}$ to 1.1 ). 

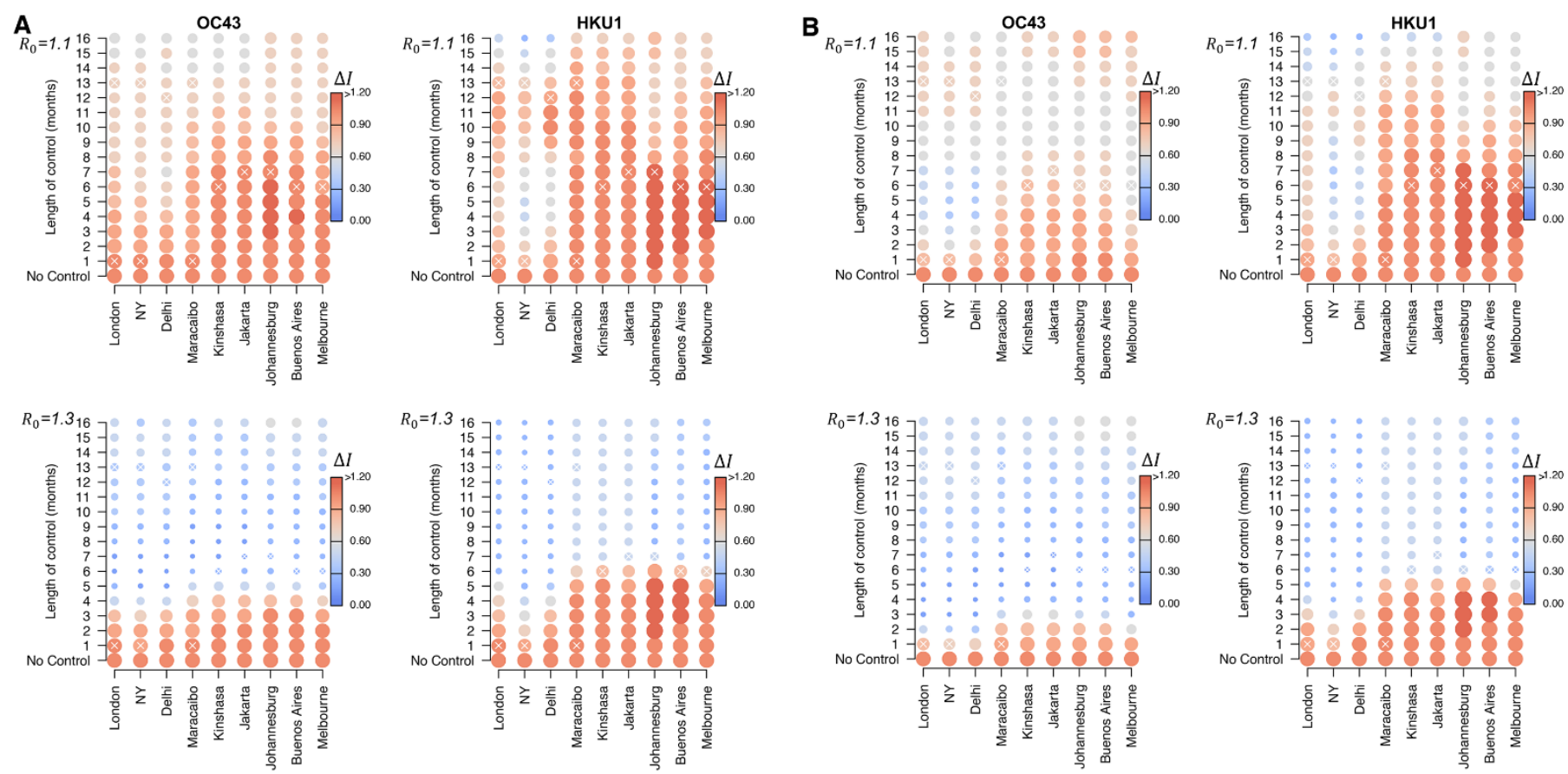

Fig. 4. Interaction between control measures and the climate. (A and B) Four scenarios representing the interaction of different climate-dependencies (OC43 and HKU1 params) with two potential control measures $\left[R_{0}=1.1\right.$ and 1.3 in the control period, occurring one month $(A)$ and six weeks (B) after pandemic start]. Size and color of the circle represents the size of peak incidence (within two years of pandemic start) relative to the no-control scenario. White crosses show month of maximum climate-driven transmission i.e., lowest specific humidity. 